\title{
The Inhibitory Effect of Validamycin A on Aspergillus flavus
}

\author{
Napasawan Plabutong, ${ }^{1,2}$ Supanuch Ekronarongchai, ${ }^{1}$ Nattarika Niwetbowornchai, ${ }^{1}$ \\ Steven W. Edwards, ${ }^{3}$ Sita Virakul, ${ }^{4}$ Direkrit Chiewchengchol, ${ }^{1,5}$ \\ and Arsa Thammahong $\mathbb{D}^{1,2}$
}

\footnotetext{
${ }^{1}$ Medical Microbiology, Interdisciplinary Program, Graduate School, Chulalongkorn University, Bangkok, Thailand

${ }^{2}$ Antimicrobial Resistance and Stewardship Research Unit, Department of Microbiology, Faculty of Medicine, Chulalongkorn University, Bangkok, Thailand

${ }^{3}$ Institute of Integrative Biology, University of Liverpool, Liverpool, UK

${ }^{4}$ Department of Microbiology, Faculty of Science, Chulalongkorn University, Bangkok, Thailand

${ }^{5}$ Translational Research in Inflammation and Immunology Research Unit, Department of Microbiology, Faculty of Medicine, Chulalongkorn University, Bangkok, Thailand
}

Correspondence should be addressed to Arsa Thammahong; arsa.t@chula.ac.th

Received 13 March 2020; Revised 8 May 2020; Accepted 3 June 2020; Published 27 June 2020

Academic Editor: Giuseppe Comi

Copyright (c) 2020 Napasawan Plabutong et al. This is an open access article distributed under the Creative Commons Attribution License, which permits unrestricted use, distribution, and reproduction in any medium, provided the original work is properly cited.

Aspergillus flavus is one of the most common isolates from patients with fungal infections. Aspergillus infection is usually treated with antifungal agents, but side effects of these agents are common. Trehalase is an essential enzyme involved in fungal metabolism, and the trehalase inhibitor, validamycin A, has been used to prevent fungal infections in agricultural products. In this study, we observed that validamycin A significantly increased trehalose levels in A. flavus conidia and delayed germination, including decreased fungal adherence. In addition, validamycin A and amphotericin B showed a combinatorial effect on A. flavus ATCC204304 and clinical isolates with high minimum inhibitory concentrations (MICs) of amphotericin B using checkerboard assays. We observed that validamycin A and amphotericin B had a synergistic effect on A. flavus strains resistant to amphotericin B. The MICs in the combination of validamycin A and amphotericin B were at $0.125 \mu \mathrm{g} / \mathrm{mL}$ and $2 \mu \mathrm{g} / \mathrm{mL}$, respectively. The FICI of validamycin A and amphotericin B of these clinical isolates was about $0.25-0.28$ with synergistic effects. No drug cytotoxicity was observed in human bronchial epithelial cells treated with validamycin A using LDH-cytotoxicity assays. In conclusion, this study demonstrated that validamycin A inhibited the growth of $A$. flavus and delayed conidial germination. Furthermore, the combined effect of validamycin A with amphotericin B increased A. flavus killing, without significant cytotoxicity to human bronchial epithelial cells. We propose that validamycin A could potentially be used in vivo as an alternative treatment for A. flavus infections.

\section{Introduction}

Aspergillus flavus is a fungus commonly found in the environment, and when it contaminates food, it produces aflatoxins, which are associated with increased risk of developing liver cancer in humans $[1,2]$. Moreover, A. flavus is an infectious fungus and can colonize organs leading to conditions such as keratitis, cutaneous infections, sinusitis, and invasive pulmonary aspergillosis [3-5]. Knowledge and understanding of the epidemiology and pathogenesis of $A$. flavus infection in humans are still very limited as there are only a few reports on A. flavus in comparison to other
Aspergillus species [6]. For example, it has been reported that A. flavus is a common cause of cutaneous infections and sinusitis in India $[4,5]$.

Initial treatment of Aspergillus invasive infections (invasive aspergillosis) begins with antifungal agents, particularly azoles. Voriconazole is a drug of choice in patients with aspergillosis $[7,8]$, but serious adverse reactions have been reported in many studies, such as transient visual disturbances, hepatotoxicity, tachyarrhythmias, and QTc interval prolongations [8]. Amphotericin B is a fungicidal polyene agent, which is an alternative, relatively cheap treatment for aspergillosis $[7,8]$, but it also has serious side 
effects (e.g., nephrotoxicity) [9]. Owing to socioeconomic status of patients and availability of this agent, the use of amphotericin B as a treatment against aspergillosis is very common in developing countries, including Thailand [10-12]. Unfortunately, recent studies have demonstrated increasing incidence of $A$. flavus clinical isolates with resistance to amphotericin B $[13,14]$.

Although patients with aspergillosis are treated with standard antifungal therapy as mentioned, evidence shows that the morbidity and mortality rates in patients with these infections are still high (up to 80\%) [15]. Therefore, the discovery of novel antifungal agents with fewer side effects is crucial for treatment of aspergillosis. Many studies have reported virulence factors and metabolic pathways that are specific to this fungus, and these could potentially be new targets for the development of antifungal agents [16, 17]. For example, trehalose is a disaccharide that is only found in bacteria, plants, insects, and invertebrates. It is composed of two glucose molecules conjugated with $\alpha, \alpha$-1, 1-glycosidic linkage, and serves as an energy source, particularly when fungi are exposed to environmental stresses such as cold, heat, and desiccation [18-20].

There are three different enzymes involved in the trehalose pathway: (a) trehalose-6-phosphate synthase (Tps1p), (b) trehalose-6-phosphate phosphatase (Tps2p), and (c) trehalase (Figure 1). Tpslp converts UDP-glucose and glucose 6-phosphate into trehalose-6-phosphate [20]. Tps2p enzyme removes phosphate from trehalose-6-phosphate to form trehalose. These enzymes in the trehalose pathway are essential for the growth of Candida albicans, Cryptococcus neoformans, and Aspergillus fumigatus [18, 21-23]. Trehalase hydrolyzes and degrades trehalose into two glucose molecules [24]. There are two types of trehalase found in Saccharomyces cerevisiae [25], which are neutral trehalase and acid trehalase (Figure 1). Neutral trehalase (Nth1p) is found in the cytosol and works at an optimum pH of $7.0[24,26]$, whereas acid trehalase (Athlp) is a cell wall-linked enzyme and works at an optimum $\mathrm{pH}$ of 5.0 [27-29]. It has been reported that the trehalose pathway is involved in the pathogenesis of fungal infections in humans (e.g., C. albicans, C. neoformans, and A. fumigatus) [19,21-23,30-32].

In previous studies, it was demonstrated that Rhizoctonia solani, a rice fungal pathogen, was inhibited by the trehalase inhibitor, validamycin A [33-35]. Validamycin A was originally isolated from Streptomyces hygroscopicus var. limoneus $[33,36,37]$, and it was shown that it inhibited branching of $R$. solani $[33,38]$. Another study found that validamycin A delayed conidial production of Fusarium culmorum [38]. However, the effectiveness of validamycin A against human fungal pathogens and its toxicity on human cells are unknown. Here, we investigated the effects of validamycin $\mathrm{A}$ alone and in combination with amphotericin $\mathrm{B}$ on the growth of A. flavus, including the cytotoxicity of validamycin A to a human cell line.

\section{Materials and Methods}

2.1. Fungal Strains, Media, and Conditions. A. flavus ATCC 204304 was cultured on Sabouraud dextrose agar (SDA,
Oxoid, Thermo Fisher Scientific) Petri-dish plates at $37^{\circ} \mathrm{C}$ for three days before harvesting $A$. flavus conidia using sterile distilled water with $0.01 \%$ Tween 80 . In brief, $5 \mathrm{~mL}$ of sterile distilled water with $0.01 \%$ Tween 80 was utilized to harvest A. flavus conidia on SDA Petri-dish plates using cell scrapers. The mixture between distilled water with Tween 80 and A. flavus conidia was filtered using Miracloth. A number of conidia were counted from the filtrate using a hemocytometer. Then, $10^{3}$ conidia were inoculated into culture media [39], i.e., glucose peptone agar (peptone $10 \mathrm{~g}$, glucose $20 \mathrm{~g}$, agar $20 \mathrm{~g}$, distilled water $1000 \mathrm{ml}$, and $\mathrm{pH} 6.8-7.0$ ), trehalose peptone agar (peptone $10 \mathrm{~g}$, trehalose $10 \mathrm{~g}$, agar $20 \mathrm{~g}$, distilled water $1000 \mathrm{ml}$, and $\mathrm{pH} 6.8-7.0$ ), and peptone agar (peptone $10 \mathrm{~g}$, agar $20 \mathrm{~g}$, distilled water $1000 \mathrm{ml}$, and $\mathrm{pH}$ $6.8-7.0$ ), incubated at $37^{\circ} \mathrm{C}$ for $2-5$ days. The radial fungal growth was measured in three biological replicates.

A. flavus clinical isolates were obtained from the Mycology Laboratory, Department of Microbiology, Faculty of Medicine, Chulalongkorn University, and King Chulalongkorn Memorial Hospital during 2019. Patient characteristics were collected from medical records/charts. Patients with invasive aspergillosis (IA) were classified as proven, probable, and possible invasive aspergillosis according to EORTC/MSG criteria [40, 41].

2.2. Trehalose Measurements. Conidia of A. flavus ATCC 204304 from SDA treated with or without $1 \mu \mathrm{g} / \mathrm{mL}$ validamycin A were collected at day 5 after incubation at $37^{\circ} \mathrm{C}$. Trehalose levels of $A$. flavus conidia were measured, as previously described [42]. In brief, $2 \times 10^{8}$ conidia in $500 \mathrm{uL}$ distilled water with Tween 80 were boiled at $100^{\circ} \mathrm{C}$ for $20 \mathrm{~min}$ and centrifuged at $11,000 \times \mathrm{g}$ for $10 \mathrm{~min}$. The supernatant was collected for trehalose measurement (with biological triplicates) using the glucose oxidase assay protocol (Sigma; GAGO20). The reaction was measured at $490 \mathrm{~nm}$ using a spectrophotometer (Lambda 1050+ UV/Vis/ NIR, PerkinElmer, USA).

2.3. Germination Assay. Conidia of A. flavus ATCC 204304 at $1 \times 10^{8}$ cells were incubated in $10 \mathrm{~mL}$ Sabouraud dextrose broth at $37^{\circ} \mathrm{C}$ in an orbital shaker at $200 \mathrm{rpm}$. The cultured broth $(500 \mu \mathrm{L})$ was used for counting percentage of germlings. The germinated conidia are counted using a microscope. At each time point, 100 conidia were counted, and the number of germinated conidia was calculated as a percentage out of total 100 conidia [43]. Each strain was cultured up to $24 \mathrm{~h}$ at $37^{\circ} \mathrm{C}$ in three biological replicates [44].

2.4. XTT Assay. XTT assays (sodium 2,3-bis (2-methoxy-4nitro-5-sulfophenyl)-5-[(phenylamino)-carbonyl]-2H-tetrazolium) were performed as described previously $[45,46]$. In brief, $10^{3}$ conidia of $A$. flavus ATCC 204304 were incubated with different culture media with or without validamycin $\mathrm{A}$ in a 96 -well plate at $37^{\circ} \mathrm{C}$ for $18 \mathrm{~h}$. XTT solution $(0.5 \mathrm{mg} / \mathrm{mL}$ in PBS $)$ was added into each well and incubated at $37^{\circ} \mathrm{C}$ for $15 \mathrm{~min}$. The plate was centrifuged, and the supernatant was collected to measure the OD at $490 \mathrm{~nm}$ using a 


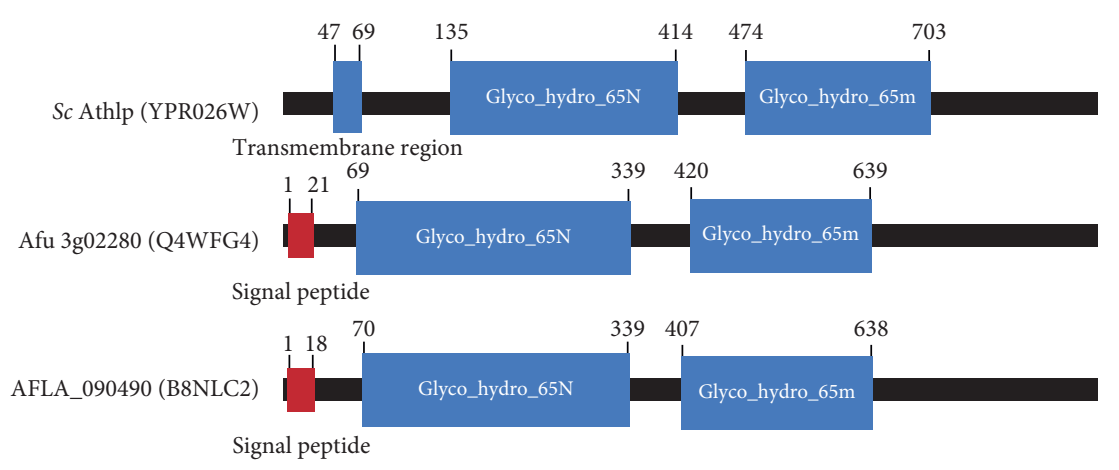

(a)

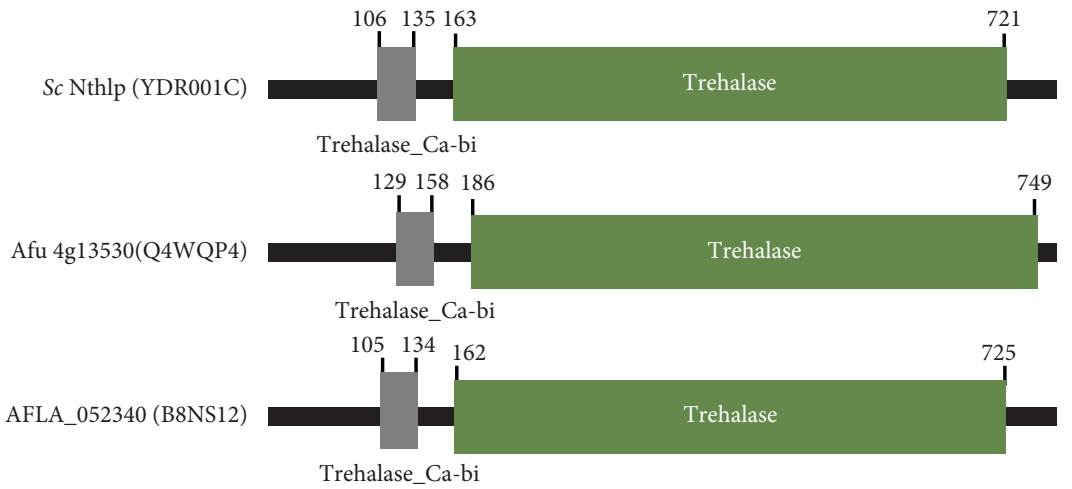

(b)

Figure 1: Aspergillus flavus possesses trehalase homologs. (a) Percentages of identity and similarity of ScAth1p (YPR026W) : AFLA_090490 (B8NLC2) and Afu3g02280 (Q4WFG4) : AFLA_090490 (B8NLC2) from BLASTp analyses are 29\% identity, 46\% similarity and 68\% identity, 81\% similarity, respectively. ScAth1p, Saccharomyces cerevisiae acid trehalase protein; Afu, Aspergillus fumigatus; AFLA, Aspergillus flavus; glycosyl hydrolase family 65 (Glyco_hydro_65N; Glyco_hydro_65m) (adapted from SMART analyses (http://smart.embl-heidelberg.de/)).(b) Percentages of identity and similarity of ScNth1p (YDR001C) : AFLA_052438 (B8NS12) and Afu4g13530 (Q4WQP4) : AFLA_052438 (B8NS12) from BLASTp analyses are 55\% identity, 69\% similarity and $81 \%$ identity, $88 \%$ similarity, respectively. ScNth1p, Saccharomyces cerevisiae neutral trehalase protein; Afu, Aspergillus fumigatus; AFLA, Aspergillus flavus; Trehalase_Ca-bi: neutral trehalase calcium-binding domain; trehalase: trehalose hydrolysis domain (adapted from SMART analyses (http://smart.embl-heidelberg.de/)).

spectrophotometer (Lambda 1050+ UV/Vis/NIR, PerkinElmer, USA).

2.5. Crystal Violet Adherence Assay. $10^{5}$ conidia per $\mathrm{mL}$ of $A$. flavus ATCC204304 were incubated in $100 \mu \mathrm{L}$ of Sabouraud dextrose broth in each well of plastic U-bottomed 96-well plates at $37^{\circ} \mathrm{C}$ for $24 \mathrm{~h}$. After washing each well twice with sterile distilled water gently, $0.1 \%$ crystal violet was utilized to stain for $10 \mathrm{~min}$. Sterile distilled water was then utilized to wash twice, and $100 \%$ ethanol was used to destain for $10 \mathrm{~min}$. Supernatants were then measured at $600 \mathrm{~nm}$ using a spectrophotometer (Lambda 1050+ UV/Vis/NIR, PerkinElmer, USA) [47].

2.6. Broth Microdilution Assay and Checkerboard Assay. The CLSI broth microdilution M38 method was performed to observe the minimum inhibitory concentrations (MICs) of amphotericin B for A. flavus ATCC 204304 and clinical isolates [48]. The additive/synergistic effect of validamycin A and amphotericin B was identified using the checkerboard assays [49]. Fractional inhibitory concentration index (FICI) was calculated for each antifungal drug, in each combination used, with the following formula [49]:

$$
\operatorname{FICA}\left(\frac{\operatorname{MIC}_{\mathrm{A}}}{\operatorname{MIC}_{\mathrm{A}+\mathrm{B}}}\right)+\operatorname{FICB}\left(\frac{\mathrm{MIC}_{\mathrm{B}}}{\operatorname{MIC}_{\mathrm{A}+\mathrm{B}}}\right)=\operatorname{FICI}
$$

FICI results were determined as follows: synergy: $<0.5$; additivity: $0.5-1$; indifference: $>1-4$; and antagonism: $>4$.

2.7. Cell Line and Culture. BEAS-2B (human bronchial epithelial cell line) (ATCC ${ }^{\circledR}$ CRL9609 $^{\mathrm{TM}}$ ) was cultured with Bronchial Epithelial Cell Growth Basal Medium (BEBM) in tissue culture flasks coated with $0.01 \mathrm{mg} / \mathrm{mL}$ fibronectin, $0.03 \mathrm{mg} / \mathrm{mL}$ bovine collagen type I, and $0.01 \mathrm{mg} / \mathrm{mL}$ bovine serum albumin (BSA). The cells were incubated at $37^{\circ} \mathrm{C}$ in a humidified environment with $5 \% \mathrm{CO}_{2}$ [50].

2.8. Cytotoxicity Assay. The cytotoxicity of validamycin A towards human epithelial cell lines was performed using a Lactate Dehydrogenase (LDH) Cytotoxicity Colorimetric Assay Kit II (BioVision Inc., CA, USA). In brief, $1 \times 10^{4}$ BEAS-2B cells were incubated with $50 \mu \mathrm{L}$ of DMEM in a 


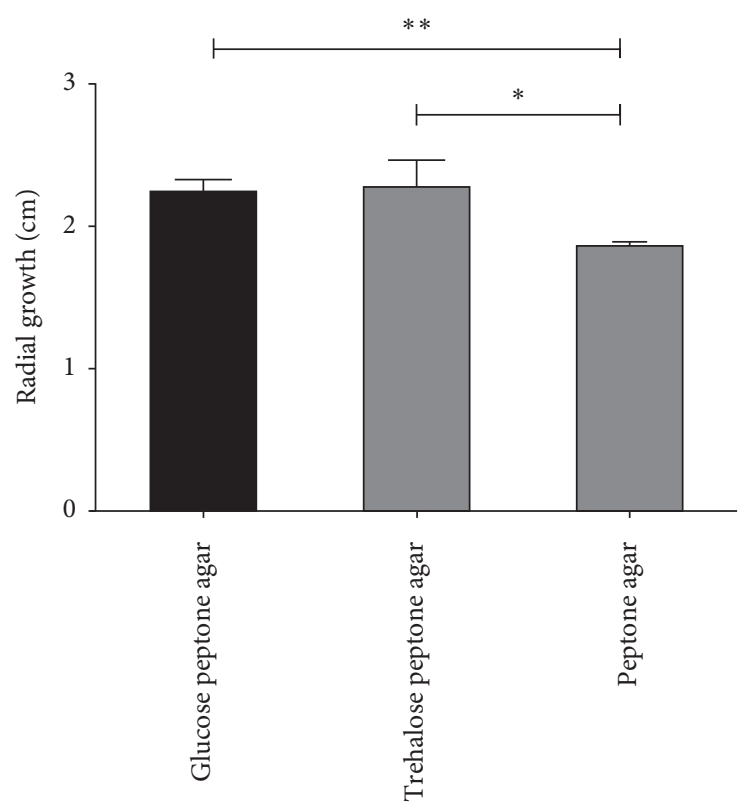

(a)

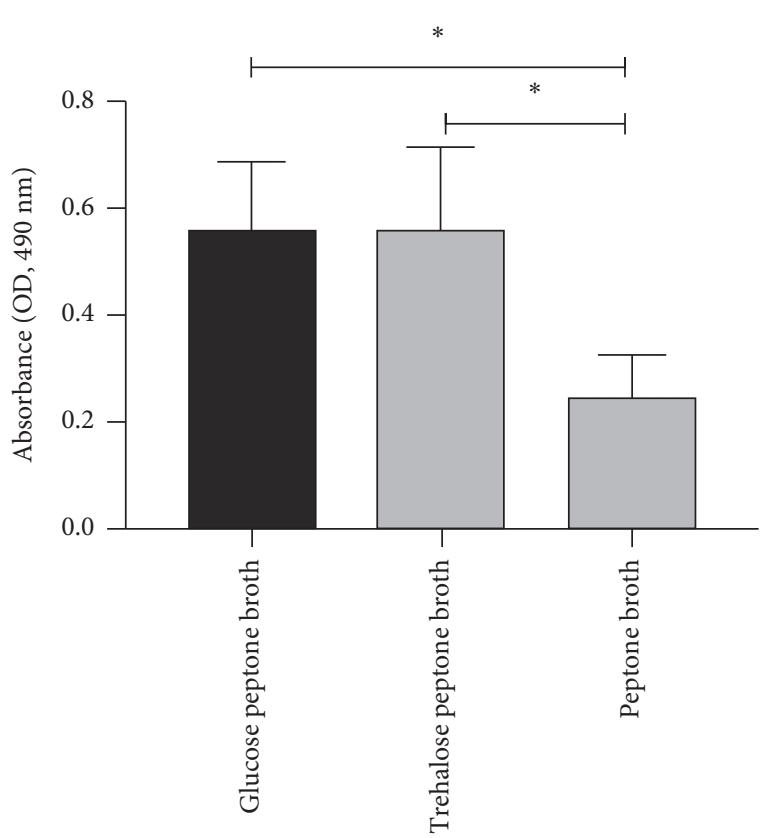

(b)

Figure 2: Aspergillus flavus utilizes trehalose as a sole carbon source similar to glucose. (a) Aspergillus flavus ATCC 204304 was incubated at $37^{\circ} \mathrm{C}$ on glucose peptone, trehalose peptone, and peptone alone media. The radial growth of these fungal growths was measured on the second day of incubation. Data are presented as means \pm SE from three biological replicates. ${ }^{*} P$ value $<0.05$; ${ }^{* *} P$ value $<0.01$ (one-way ANOVA with post hoc Bonferroni's test). (b) Aspergillus flavus ATCC 204304 was incubated at $37^{\circ} \mathrm{C}$ on glucose peptone, trehalose peptone, and peptone alone liquid media for 24 hours, and viability tests using XTT assays were performed. Data are presented as means \pm SE from three biological replicates. ${ }^{*} P$ value $<0.05$ (one-way ANOVA with post hoc Bonferroni's test).

precoated 96-well plate, and then validamycin A was added at different concentrations $(1 \mu \mathrm{g} / \mathrm{mL}-1 \mathrm{mg} / \mathrm{mL}$, final concentration). LDH reaction mixture was added, and the cells were incubated at $37^{\circ} \mathrm{C}$ for $30 \mathrm{~min}$. LDH released from the cells was measured at $450 \mathrm{~nm}$ using a spectrophotometer. The percentage of cytotoxicity was calculated using the following formula:

$$
\operatorname{Cytotoxicity~}(\%)=\frac{(\text { test sample }- \text { low control }) \times 100}{(\text { high control }- \text { low control })} .
$$

High control is cells with lysis buffer, while low control is cells alone as a background.

2.9. Statistical Analysis. All statistical analyses were conducted with Prism 8 software (GraphPad Software, Inc., San Diego, CA). Comparison between groups was performed with unpaired two-tailed Student's $t$-tests for two data groups and one-way ANOVA tests with post hoc Bonferroni's multiple comparison tests for more than two data groups. Error bars represent standard errors of the means. Significant differences were considered when $P$ value $<0.05$.

2.10. Ethical Statement. This study was approved by the Institutional Review Board (IRB no. 546/60), Faculty of Medicine, Chulalongkorn University, Bangkok, Thailand.

\section{Results}

3.1. Trehalase Homologs in Aspergillus flavus. To identify trehalase enzyme homologs in A. flavus, a BLASTp search was performed on $S$. cerevisiae and A. fumigatus and compared with A. flavus. The protein data from the FungiDB database and Simple Modular Architecture Research Tool (SMART) were used to compare putative protein domains among trehalase enzymes from $S$. cerevisiae $(S c)$, A. fumigatus (Afu), and A. flavus (AFLA) (database: https://fungidb. org and http://smart.embl-heidelberg.de).

The results showed that AFLA_090490 protein, containing one signal peptide at positions $1-18$ and two O-glycosyl hydrolase domains (EC 3.2.1) at positions 70-339 and 407-638, was similar to acid trehalase of S. cerevisiae and A. fumigatus (Figure 1(a)). AFLA_052430 protein, containing a neutral trehalase calcium-binding domain at positions 105-134 and an O-glycosyl hydrolase domain (EC 3.2.1) at positions $162-725$, was similar to neutral trehalase of S. cerevisiae and A. fumigatus (Figure 1(b)). Our findings suggest that $A$. flavus has both acid and neutral trehalases, as seen in S. cerevisiae and A. fumigatus.

Next, we investigated the ability of $A$. flavus to utilize trehalose as a sole carbon source. The result showed that growth and viability of $A$. flavus on glucose peptone media and trehalose peptone media were similar (Figures 2(a) and 2(b)). This finding supports the idea that A. flavus utilizes trehalose as a sole carbon source and implies that it degrades extracellular trehalose into glucose for its growth. 
3.2. Growth Inhibition and Decreased Fungal Adherence of Aspergillus flavus by Validamycin A. To observe the inhibitory effect of validamycin A on A. flavus ATCC204304, broth microdilution and XTT assays were performed. The results showed that the minimal inhibition concentration (MIC) of validamycin A against A. flavus was $1 \mu \mathrm{g} / \mathrm{mL}$ (Table 1), and the viability of A. flavus ATCC204304 after validamycin A treatment at this concentration was significantly decreased when compared with $0.5 \mu \mathrm{g} / \mathrm{mL}$ of validamycin A, $0.25 \mu \mathrm{g} / \mathrm{mL}$ of amphotericin $\mathrm{B}$, and the control group (Figure 3).

Next, A. flavus ATCC204304 was cultured and treated with or without 0.5 and $1 \mu \mathrm{g} / \mathrm{mL}$ of validamycin $\mathrm{A}$, and trehalose levels in the conidia were measured. The results demonstrated that conidia collected from A. flavus treated with validamycin A showed significantly higher levels of trehalose than the control (untreated) group, suggesting that validamycin A inhibited trehalase enzymes in the conidia of A. flavus (Figure 4(a)). In addition, the rate of conidial germination was investigated in A. flavus conidia treated with $1 \mu \mathrm{g} / \mathrm{mL}$ of validamycin $\mathrm{A}$. The results showed that validamycin A significantly delayed conidial germination of A. flavus ATCC204304 particularly at 10 and $12 \mathrm{~h}$ (Figure 4(b)). These data suggest that validamycin A delays conidial germination of $A$. flavus via inhibition of trehalase enzymes.

To observe the effect of validamycin A on exopolysaccharides of $A$. flavus, the crystal violet adherence assays were performed. We observed that $1 \mu \mathrm{g} / \mathrm{mL}$ of validamycin A decreased the adherence property of A. flavus ATCC204304 (Figure 4(c)). These data suggest that validamycin A affects the fungal adherence of A. flavus.

\subsection{Synergistic Effects of Validamycin A and Amphotericin B} on Aspergillus flavus Clinical Isolates. Antifungal susceptibility tests of A. flavus ATCC204304 were performed according to the CLSI broth microdilution method (CLSI M38, 2017). The results demonstrated that the MIC of validamycin $\mathrm{A}$ and amphotericin $\mathrm{B}$ alone against $A$. flavus ATCC204304 was 1 and $4 \mu \mathrm{g} / \mathrm{mL}$, respectively (Table 1 ). Furthermore, the fractional inhibitory concentration index (FICI) was 0.625 with the concentrations of validamycin $\mathrm{A}$ and amphotericin B at $0.125 \mu \mathrm{g} / \mathrm{mL}$ and $2 \mu \mathrm{g} / \mathrm{mL}$, respectively (Table 1). This finding suggests that validamycin A and amphotericin B have an additive effect on A. flavus ATCC204304.

To confirm the combinative effects of validamycin A and amphotericin B, A. flavus clinical isolates $(n=3)$ with high MICs of amphotericin B $(>4 \mu \mathrm{g} / \mathrm{mL})$ (Table 1) were chosen to perform checkerboard assays. Interestingly, the FICI was $0.25-0.28$, suggesting a synergistic effect between these two drugs on these clinical isolates (Table 1).

3.4. No Cytotoxicity of Validamycin A to Human Bronchial Epithelial Cells. Human bronchial epithelial cells, BEAS-2B, were treated with or without validamycin A including amphotericin $\mathrm{B}$ at different concentrations. The results demonstrated that $0.125,0.5$, and $1 \mu \mathrm{g} / \mathrm{mL}$ of validamycin $\mathrm{A}$,
1 and $2 \mu \mathrm{g} / \mathrm{mL}$ of amphotericin $\mathrm{B}$, and a combination of these two drug concentrations of $0.125 \mu \mathrm{g} / \mathrm{mL}$ of validamycin A and $2 \mu \mathrm{g} / \mathrm{mL}$ of amphotericin B showed no significant cytotoxicity to human bronchial epithelial cells (Figure 5).

\section{Discussion}

The trehalose pathway is a major mechanism for growth and metabolism of many fungi; however, the presence of trehalase enzymes in many of these fungi is still unknown $[19,21-23,30-32]$. Validamycin A is a trehalase enzyme inhibitor produced by Streptomyces hygroscopicus and is used for fungal inhibition in plants and insects $[33,36,37,51,52]$. From many previous reports, in plants and insects, the effect of validamycin $A$ is to inhibit trehalase activity in their cells [53-56]. In a rice fungal pathogen, Rhizoctonia solani, validamycin A was shown to inhibit trehalase activity but not cellulase, pectinase, chitinase, amylase, or glucosidases [57]. Additionally, validamycin A also inhibited the growth of Rhizoctonia solani and Fusarium culmorum $[33,38]$. However, there are only few studies demonstrating the effects of validamycin A on human fungal pathogens [58]. From our study, we observed that a human fungal pathogen, A. flavus, had two trehalase enzymes that shared similar conserved domains and possessed high similarity and identity to Saccharomyces cerevisiae and Aspergillus fumigatus (Figures 1(a) and 1(b)), including Rhizoctonia solani and Candida albicans (Figures S1(a)and S1(b)). Therefore, we hypothesize that validamycin A may inhibit trehalase enzyme activity in A. flavus similar to previous reports $[33,38,57]$.

In this study, we investigated the presence of trehalase enzymes and the effect of the trehalase inhibitor, validamycin $\mathrm{A}$, on the growth of a common pathogenic fungus in humans, A. flavus. The results showed that A. flavus possesses trehalase homologs and grows on trehalose peptone media, similar to growth on glucose peptone media (Figures 2(a) and 2(b)). These findings imply that A. flavus utilizes trehalase enzymes to degrade trehalose for use as a carbon source and energy. In addition, we observed inhibitory effects of validamycin A on the growth of A. flavus (Figure 3). This finding suggests that trehalase activity is required for A. flavus growth. However, direct evidence, such as genetic approaches (e.g., generating trehalase genedeletion mutants) to support the importance of trehalase, is needed to confirm this observation.

In a previous study, it was found that validamycin $\mathrm{A}$ increased trehalose levels in a pathogenic fungus, C. albicans [58]. This result is similar to our findings that showed an increase in trehalose levels of $A$. flavus conidia after validamycin A treatment (Figure 4(a)). However, further trehalase activity assay using high-performance liquid chromatography (HPLC) is also necessary to confirm the effect of validamycin A against trehalase enzymes in $A$. flavus. As the trehalose pathway is crucial in the early stages of conidial germination [18, 19, 47, 59], we further investigated the effect of validamycin A on conidial germination of A. flavus. Expectedly, validamycin A significantly delayed 


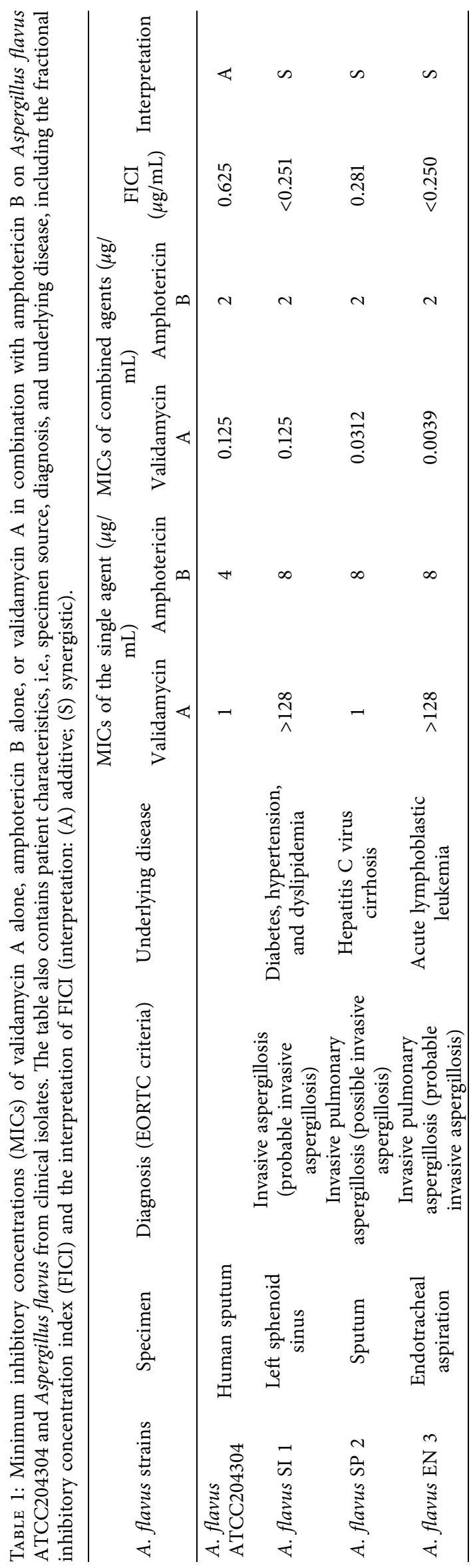




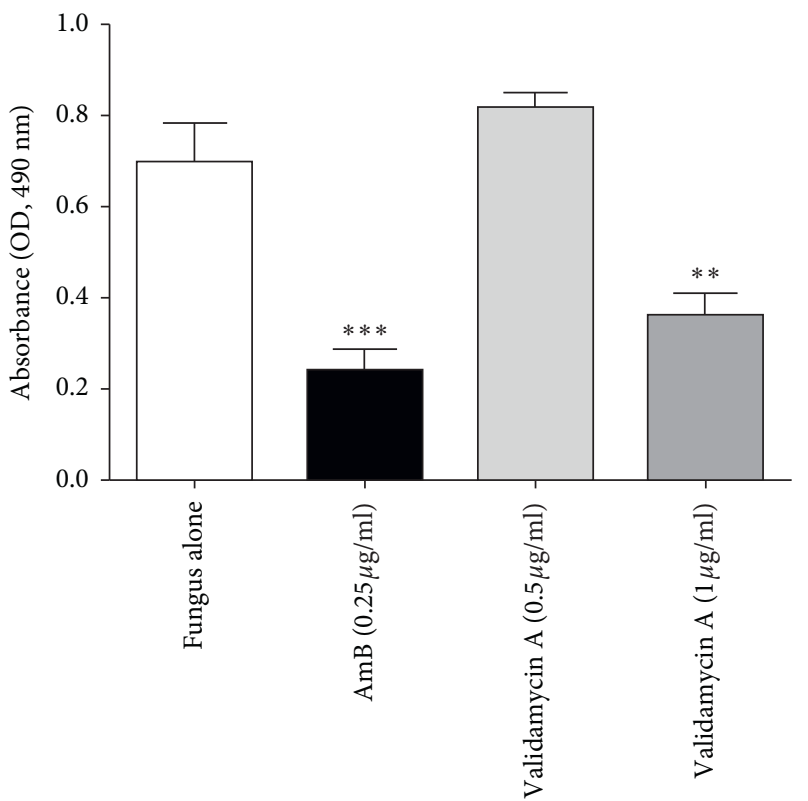

Figure 3: Validamycin A inhibits the growth of Aspergillus flavus. Aspergillus flavus ATCC204304 was cultured at $37^{\circ} \mathrm{C}$ in RPMI media in a 24 -well plate for 18 hours. Fungal viability was measured by XTT assays at $490 \mathrm{~nm}$. Amp, amphotericin B at $0.25 \mu \mathrm{g} / \mathrm{mL}$. Data are presented as means \pm SE from three biological replicates. ${ }^{* *} P$ value $<0.01$; ${ }^{* * *} P$ value $<0.001$ (one-way ANOVA with post hoc Bonferroni's test compared to fungus alone).

conidial germination of A. flavus (Figure 4(b)). Therefore, these observations suggest that the inhibition of trehalase enzymes depletes the source of energy and the growth for $A$. flavus. Nonetheless, we observed that conidial germination, in the presence of validamycin A, was not different from the untreated group at 24-hour incubation. This result suggests that $A$. flavus could probably increase conidial germination by alternative pathways following trehalase inhibition (e.g., mannitol pathway) [60, 61]. A wide variety of different media is still necessary to further investigate the trehalose phenotypes in A. flavus.

In addition, this study further investigated the combinative effect between validamycin $\mathrm{A}$ and amphotericin $\mathrm{B}$ on A. flavus ATCC204304, which is a standard strain for the antifungal susceptibility test. The result demonstrated that these two drugs showed an additive effect on growth inhibition of A. flavus. Interestingly, the combination of these drugs had a synergistic effect on A. flavus clinical isolates with high MICs of amphotericin B. Although the cutoff value of MIC for amphotericin B resistance in A. flavus was unknown, Barchiesi et al. suggested that MIC of amphotericin $B \geq 2 \mu \mathrm{g} /$ $\mathrm{mL}$ should be considered as a resistant strain $[48,62]$.

Trehalose pathway is clearly associated with cell wall components, including chitin and beta-glucan, as shown in many previous reports $[18,19,42,47]$. Disturbance in substrates of trehalose or enzymes or proteins associated with the trehalose pathway in Aspergillus fumigatus would lead to changes in the cell wall components and structure $[18,19,42,47]$. Furthermore, trehalose level and proteins associated with the trehalose pathway may affect exopolysaccharide galactosaminogalactans (GAGs), which are important for fungal adherence and biofilm formation, as shown in A. fumigatus previous reports [42, 47]. In this study, we also observed that validamycin A decreased fungal adherence (Figure 4(c)). These data imply that the structure or components of exopolysaccharide GAGs may be affected by validamycin $\mathrm{A}$.

Besides, trehalase enzymes in many eukaryotic organisms may play important roles in carbon metabolism, chitin biosynthesis, and stress tolerance, i.e., sucrose and trehalose homeostasis in Arabidopsis thaliana and Phaseolus vulgaris, regulation of chitin biosynthesis in insects, and carbon partitioning in many plants [63-70]. Therefore, we hypothesize that inhibition of the trehalase enzyme via validamycin A may change the structure and components of the fungal cell wall and exopolysaccharide through changes in the carbon metabolism of $A$. flavus leading to increased permeability and synergistic effects of amphotericin B against $A$. flavus in the presence of validamycin A. However, further studies of cell wall/GAG structures via the electron microscope and cell wall/GAG components through HPLC, including RNA sequencing and metabolomic analyses, are necessary to decipher the effect of validamycin A on A. flavus $[18,47]$.

Additionally, MICs of validamycin A in each A. flavus clinical isolate were varied. This variation of MICS of validamycin A in these clinical isolates is probably due to the difference in the cell wall/GAG structure and components of each strain (e.g., glucan or chitin), as a previous study showed that amphotericin B-resistant A. flavus contained higher $(1,3)-\beta$-D-glucan in their cell wall than the sensitive strains [71]. Furthermore, previous studies suggest that some clinical isolates of $A$. fumigatus had different phenotypes including cell wall components and virulence $[72,73]$.

We further characterized these clinical isolates and observed that the growth rate and conidial trehalose levels 


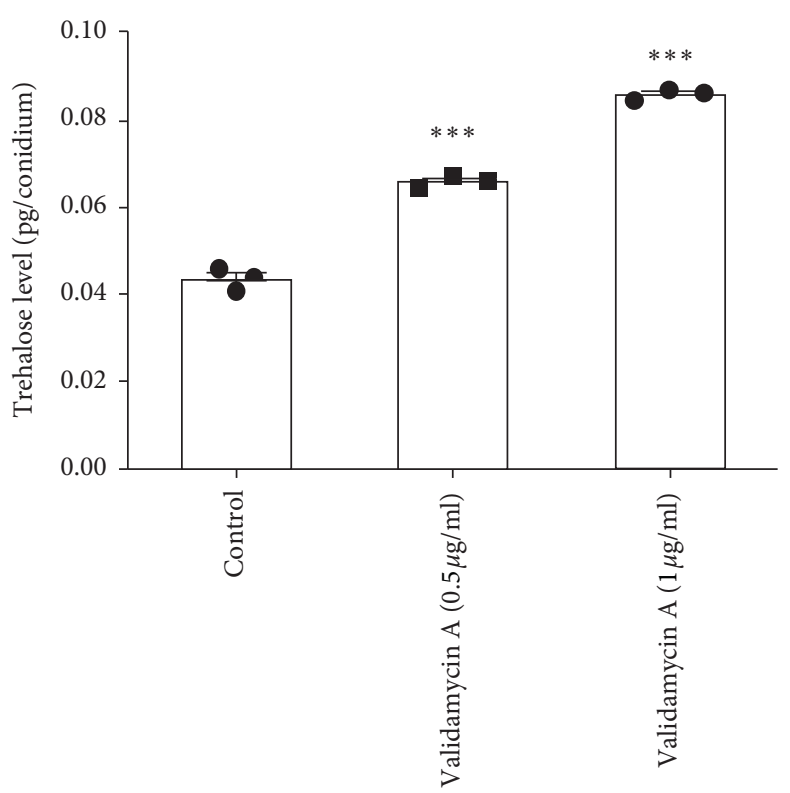

(a)

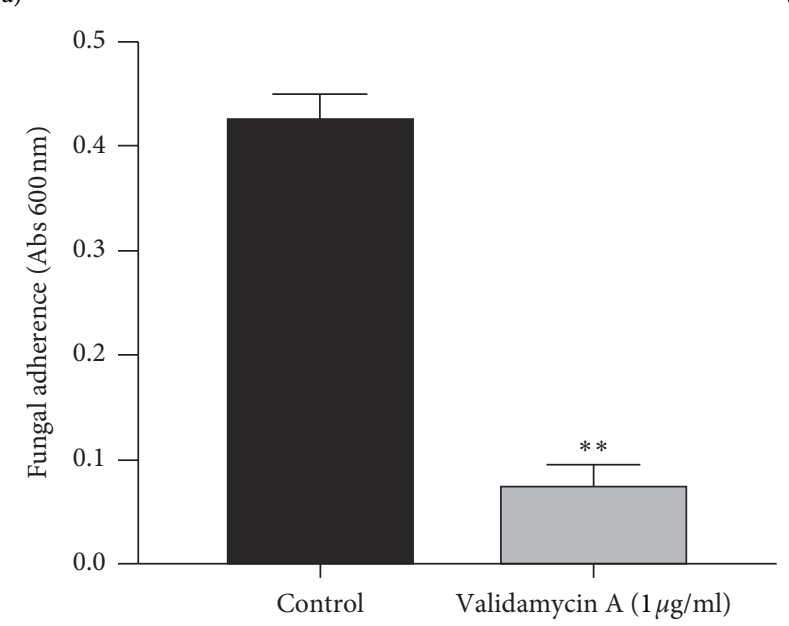

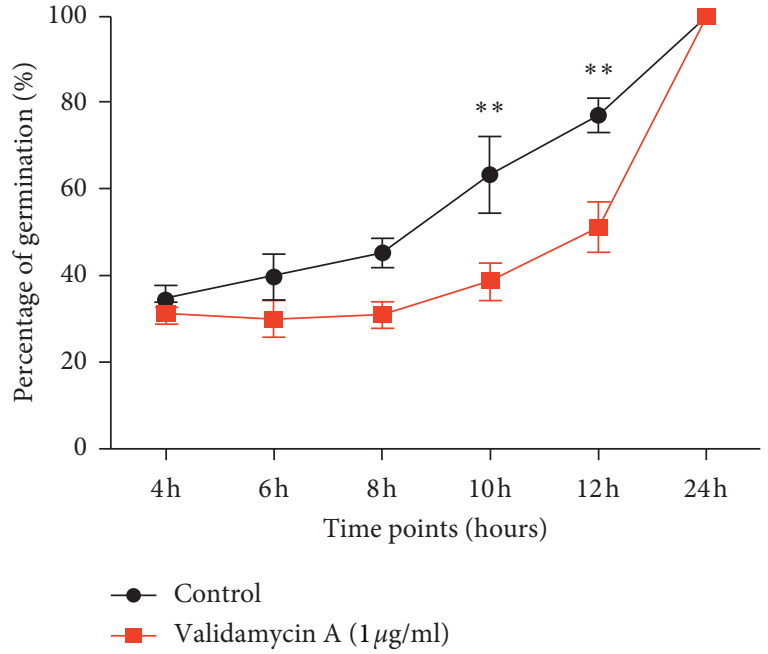

(b)

(c)

Figure 4: Validamycin A increases trehalose levels in Aspergillus flavus conidia with delayed conidial germination and decreased fungal adherence. (a) Aspergillus flavus ATCC 204304 was cultured at $37^{\circ} \mathrm{C}$ on Sabouraud dextrose agar for five days with or without $1 \mu \mathrm{g} / \mathrm{mL}$ validamycin A. Trehalose assays were performed to measure trehalose levels in the conidia using glucose oxidase assays. Data are presented as means \pm SE from three biological replicates. ${ }^{* * *} P$ value $<0.001$ (unpaired two-tailed Student's $t$-test compared with the control). (b) Aspergillus flavus ATCC 204304 was cultured at $37^{\circ} \mathrm{C}$ in Sabouraud dextrose broth with or without $1 \mu \mathrm{g} / \mathrm{mL}$ validamycin A in an orbital shaker at $200 \mathrm{rpm}$. Conidial germination at each time point was counted and calculated. Data are presented as means \pm SE from three biological replicates. ${ }^{* *} P$ value $<0.01$ (unpaired two-tailed Student's $t$-test compared with the control). (c) Aspergillus flavus ATCC 204304 was cultured at $37^{\circ} \mathrm{C}$ in Sabouraud dextrose broth with or without $1 \mu \mathrm{g} / \mathrm{mL}$ validamycin A in 96 -well plates for 24 hours, and crystal violet adherence assays were performed. Data are presented as means \pm SE from three biological replicates. ${ }^{* *} P$ value $<0.01$ (unpaired two-tailed Student's $t$-test compared with the control).

showed no difference from A. flavus ATCC204304 (Figures S2(a) and S2(b)). However, these isolates possessed different fungal adherence properties (Figure S2(c)). Different exopolysaccharide components and/or structure of these isolates may lead to decreased permeability of amphotericin B and validamycin A into the fungal cell membrane and cytoplasm affecting MICs in each clinical isolate. Nonetheless, the cell wall/GAG structure and components of these clinical isolates need to be further studied. Moreover, more clinical isolates and animal models are also necessary to confirm synergistic effects between validamycin $\mathrm{A}$ and amphotericin $\mathrm{B}$.

Cytotoxicity of validamycin A was tested in our study, and the result demonstrated that validamycin $\mathrm{A}$ at concentrations showing synergistic effects on A. flavus had no cytotoxicity on human bronchial epithelial cells (Figure 5). Nevertheless, different human cell lines together with different concentrations of validamycin A and amphotericin B are still needed to be further investigated for the cytotoxicity. In addition, in vivo studies are required as acute toxicity was 


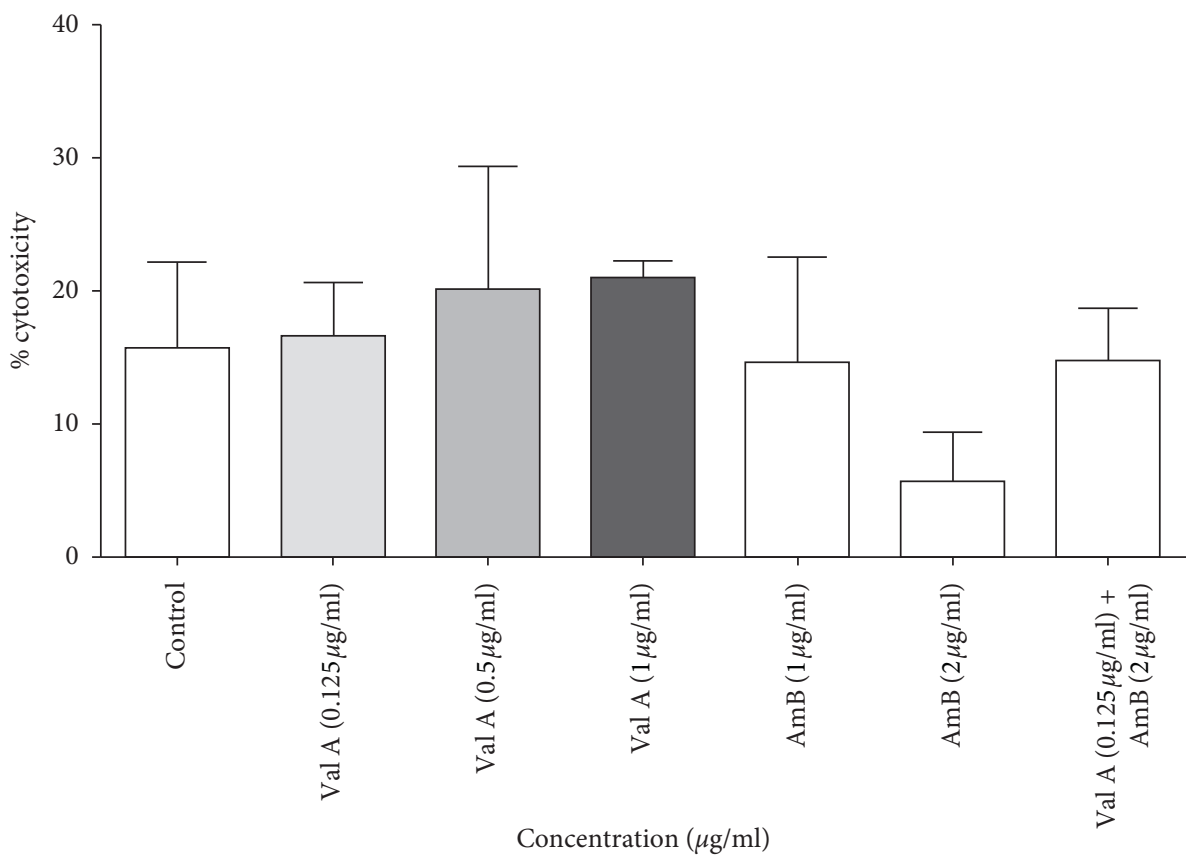

Figure 5: Validamycin A and the combination of validamycin A and amphotericin B have no cytotoxic effect on human bronchial epithelial cells. The cytotoxicity test was performed to observe the toxicity of validamycin A and amphotericin B on BEAS-2B cells using Lactate Dehydrogenase (LDH) Cytotoxicity Colorimetric Assay Kit II. Cell cultures were incubated at $37^{\circ} \mathrm{C}$ in a humidified environment containing $95 \%$ air and $5 \% \mathrm{CO}_{2}$. After 24 hours, $\mathrm{LDH}$ reaction mixture was added $(25 \mu \mathrm{l})$ and incubated at $37^{\circ} \mathrm{C}$ for 30 minutes. Then, ODs were measured at $450 \mathrm{~nm}$ using a spectrophotometer. Data are presented as means \pm SE from three biological replicates. No significant difference was observed (one-way ANOVA with post hoc Bonferroni's test).

found in rodents at very high doses of validamycin A (https:// pubchem.ncbi.nlm.nih.gov/compound/Validamycin-A). For future in vivo survival studies, different concentrations of validamycin A, i.e., 0.125 and $1 \mu \mathrm{g} / \mathrm{mL}$ with or without the combination of amphotericin B, and different routes of administration, e.g., oral gavage, intraperitoneal route, or intravenous route, are necessary to be further investigated.

In conclusion, this study demonstrated that validamycin A delayed conidial germination and inhibited the growth of $A$. flavus. Moreover, a combination between validamycin $A$ and amphotericin $B$ showed a synergistic effect on amphotericin B-resistant A. flavus clinical isolates. The cytotoxicity of validamycin A to human bronchial epithelial cells was not observed in our study. Therefore, we propose that validamycin A could potentially be used as adjunctive therapy in patients with A. flavus infection, particularly those who are infected with amphotericin B-resistant strains.

\section{Data Availability}

All data used to support the findings of this study are included within the article, and the raw data for each figure are available from the corresponding author upon request.

\section{Conflicts of Interest}

The authors declare that there are no conflicts of interest regarding the publication of this article.

\section{Acknowledgments}

The authors would like to thank the Department of Microbiology and Department of Medicine, Faculty of Medicine, Chulalongkorn University, and King Chulalongkorn Memorial Hospital for their support, especially Dr. Ariya Chindamporn, Dr. Asada Leelahavanichkul, and Dr. Nattiya Hirankarn. AT would like to thank Research Affairs, Faculty of Medicine, Chulalongkorn University, for English editing service. This research was supported by Ratchadapisek Sompotch Fund, Faculty of Medicine, Chulalongkorn University (grant no. RA61/045).

\section{Supplementary Materials}

Figure S1: Aspergillus flavus shares similar trehalase enzymes with Rhizoctonia solani and Candida albicans. (a) Percentages of identity and similarity of AFLA_090490 (B8NLC2): $R$. solani AGM46811.1 (R4VJL2) and AFLA_090490 (B8NLC2): C. albicans SC5314 acid trehalase (Q5AAU5) from BLASTp analyses are 31\% identity, 47\% similarity and $32 \%$ identity, $48 \%$ similarity, respectively. AFLA, Aspergillus flavus; glycosyl hydrolase family 65 (Glyco_hydro_65N; Glyco_hydro_65m); trehalase: trehalose hydrolysis domain (adapted from SMART analyses). (b) Percentages of identity and similarity of AFLA_052438 (B8NS12): $R$. solani AGM46812.1 (R4VM92) and AFLA_052438 (B8NS12):C. albicans P78042 neutral trehalase from BLASTp analyses are 55\% identity, $70 \%$ 
similarity and $55 \%$ identity, $71 \%$ similarity, respectively. AFLA, Aspergillus flavus; Trehalase_Ca-bi, neutral trehalase calcium-binding domain; trehalase: trehalose hydrolysis domain (adapted from SMART analyses). Figure S2: different Aspergillus flavus isolates show no difference in the radial growth rate and conidial trehalose levels but possess different fungal adherence properties. (a) Aspergillus flavus ATCC 204304 and three clinical isolates were incubated at $37^{\circ} \mathrm{C}$ on glucose media. The radial growth of these fungal growths was measured on the third day of incubation. Data are presented as means \pm SE from three biological replicates. No significant difference was observed (one-way ANOVA with post hoc Bonferroni's test). (b) Aspergillus flavus ATCC-204304 and three clinical isolates were cultured at $37^{\circ} \mathrm{C}$ on Sabouraud dextrose agar for five days with or without $1 \mu \mathrm{g} / \mathrm{mL}$ validamycin A. Trehalose assays were performed to measure trehalose levels in the conidia using glucose oxidase assays. Data are presented as means $\pm \mathrm{SE}$ from three biological replicates. No significant difference was observed (one-way ANOVA with post hoc Bonferroni's test). (c) Aspergillus flavus ATCC 204304 and three clinical isolates were cultured at $37^{\circ} \mathrm{C}$ in Sabouraud dextrose broth with or without $1 \mu \mathrm{g} / \mathrm{mL}$ validamycin A in 96-well plates for 24 hours, and crystal violet adherence assays were performed. Data are presented as means $\pm \mathrm{SE}$ from three biological replicates. ${ }^{*} P$ value $<0.05 ;{ }^{* *} P$ value $<0.01$ (one-way ANOVA with post hoc Bonferroni's test compared to the ATCC-204304 strain). (Supplementary Materials)

\section{References}

[1] I. C. Hsu, R. A. Metcalf, T. Sun, J. A. Welsh, N. J. Wang, and C. C. Harris, "Mutational hot spot in the p53 gene in human hepatocellular carcinomas," Nature, vol. 350, no. 6317, pp. 427-428, 1991.

[2] W. Hu, "The major lipid peroxidation product, trans-4-hydroxy-2-nonenal, preferentially forms DNA adducts at codon 249 of human p53 gene, a unique mutational hotspot in hepatocellular carcinoma," Carcinogenesis, vol. 23, no. 11, pp. 1781-1789, 2002.

[3] J.-A. H. van Burik, R. Colven, and D. H. Spach, "Cutaneous aspergillosis," Journal of Clinical Microbiology, vol. 36, no. 11, pp. 3115-3121, 1998.

[4] M. T. Hedayati, "Aspergillus flavus: human pathogen, allergen and mycotoxin producer," Microbiology, vol. 153, no. Pt 6, pp. 1677-1692, 2007.

[5] A. C. Pasqualotto, "Differences in pathogenicity and clinical syndromes due to Aspergillus fumigatus and Aspergillus flavus," Medical Mycology, vol. 47, no. s1, pp. S261-S270, 2009.

[6] A. Chakrabarti, S. S. Chatterjee, and M. Shivaprakash, "Overview of opportunistic fungal infections in India," Nippon Ishinkin Gakkai Zasshi, vol. 49, no. 3, pp. 165-172, 2008.

[7] S. Krishnan, E. K. Manavathu, and P. H. Chandrasekar, "Aspergillus flavus: an emerging non-fumigatus Aspergillusspecies of significance," Mycoses, vol. 52, no. 3, pp. 206222, 2009.

[8] T. F. Patterson, G. R. Thompson, D. W. Denning et al., "Practice guidelines for the diagnosis and management of aspergillosis: 2016 update by the infectious diseases society of America," Clinical Infectious Diseases, vol. 63, no. 4, pp. e1-e60, 2016.
[9] R. Laniado-Laborín and M. N. Cabrales-Vargas, "Amphotericin B: side effects and toxicity," Revista Iberoamericana de Micología, vol. 26, no. 4, pp. 223-227, 2009.

[10] S. Kiertiburanakul, C. Thibbadee, and P. Santanirand, "Invasive aspergillosis in a tertiary-care hospital in Thailand," Journal of the Medical Association of Thailand Chotmaihet Thangphaet, vol. 90, no. 5, pp. 895-902, 2007.

[11] A. Thammahong, "Invasive Aspergillus infections in a Thai tertiary-care hospital during 2006-2011," Advances in Microbiology, vol. 5, no. 5, p. 9, 2015.

[12] A. Chakrabarti, S. S. Chatterjee, A. Das, and M. R. Shivaprakash, "Invasive aspergillosis in developing countries," Medical Mycology, vol. 49, no. S1, pp. S35-S47, 2011.

[13] J. W. Baddley, K. A. Marr, D. R. Andes et al., "Patterns of susceptibility of Aspergillus isolates recovered from patients enrolled in the transplant-associated infection surveillance Network," Journal of Clinical Microbiology, vol. 47, no. 10, pp. 3271-3275, 2009.

[14] S. M. Rudramurthy, "Invasive aspergillosis by Aspergillus flavus: epidemiology, diagnosis, antifungal resistance, and management," Journal of Fungi, vol. 5, no. 3, 2019.

[15] J. Perlroth, B. Choi, and B. Spellberg, "Nosocomial fungal infections: epidemiology, diagnosis, and treatment," Medical Mycology, vol. 45, no. 4, pp. 321-346, 2007.

[16] K. Gauwerky, C. Borelli, and H. C. Korting, "Targeting virulence: a new paradigm for antifungals," Drug Discovery Today, vol. 14, no. 3-4, pp. 214-222, 2009.

[17] J. R. Perfect, "Fungal virulence genes as targets for antifungal chemotherapy," Antimicrobial Agents and Chemotherapy, vol. 40, no. 7, pp. 1577-1583, 1996.

[18] S. Puttikamonkul, S. D Willger, N Grahl et al., "Trehalose 6phosphate phosphatase is required for cell wall integrity and fungal virulence but not trehalose biosynthesis in the human fungal pathogen Aspergillus fumigatus," Molecular Microbiology, vol. 77, no. 4, pp. 891-911, 2010.

[19] N. Al-Bader, G. Vanier, H. Liu et al., "Role of trehalose biosynthesis in Aspergillus fumigatus development, stress response, and virulence," Infection and Immunity, vol. 78, no. 7, pp. 3007-3018, 2010.

[20] A. Thammahong, "Central role of the trehalose biosynthesis pathway in the pathogenesis of human fungal infections: opportunities and challenges for therapeutic development," $M i$ crobiology and Molecular Biology Reviews, vol. 81, no. 2, 2017.

[21] E. W. Petzold, U. Himmelreich, E. Mylonakis et al., "Characterization and regulation of the trehalose synthesis pathway and its importance in the pathogenicity of Cryptococcus neoformans," Infection and Immunity, vol. 74, no. 10, pp. 5877-5887, 2006.

[22] O. Zaragoza, "Disruption in Candida albicans of the TPS2 gene encoding trehalose-6-phosphate phosphatase affects cell integrity and decreases infectivity," Microbiology, vol. 148, no. Pt 5, pp. 1281-1290, 2002.

[23] P. Van Dijck, L. De Rop, K. Szlufcik, E. Van Ael, and J. M. Thevelein, "Disruption of the Candida albicans TPS2 gene encoding trehalose-6-phosphate phosphatase decreases infectivity without affecting hypha formation," Infection and Immunity, vol. 70, no. 4, pp. 1772-1782, 2002.

[24] Y. Zhou, N. O. Keyhani, Y. Zhang et al., "Dissection of the contributions of cyclophilin genes to development and virulence in a fungal insect pathogen," Environmental Microbiology, vol. 18, no. 11, pp. 3812-3826, 2016.

[25] I. Kienle, M. U. Burgert, and H. Holzer, "Assay of trehalose with acid trehalase purified from Saccharomyces cerevisiae," Yeast, vol. 9, no. 6, pp. 607-611, 1993. 
[26] H. Zähringer, M. Burgert, H. Holzer, and S. Nwaka, "Neutral trehalase Nth1p of Saccharomyces cerevisiae encoded by the NTH1 gene is a multiple stress responsive protein," FEBS Letters, vol. 412, no. 3, pp. 615-620, 1997.

[27] S. Nwaka, B. Mechler, and H. Holzer, "Deletion of the ATH1 gene in Saccharomyces cerevisiae prevents growth on trehalose," FEBS Letters, vol. 386, no. 2-3, pp. 235-238, 1996.

[28] Y. Pedreño, S. Maicas, J.-C. Argüelles, R. Sentandreu, and E. Valentin, "The ATC1 Gene encodes a cell wall-linked acid trehalase required for growth on trehalose in Candida albicans," Journal of Biological Chemistry, vol. 279, no. 39, pp. 40852-40860, 2004.

[29] M. Jules, V. Guillou, J. François, and J.-L. Parrou, "Two distinct pathways for trehalose assimilation in the yeast Saccharomyces cerevisiae," Applied and Environmental Microbiology, vol. 70, no. 5, pp. 2771-2778, 2004.

[30] A. D. Elbein, Y. T. Pan, I. Pastuszak, and D. Carroll, "New insights on trehalose: a multifunctional molecule," Glycobiology, vol. 13, no. 4, pp. 17R-27R, 2003.

[31] A. D. Elbein, "The metabolism of $\alpha, \alpha$-trehalose," Advances in Carbohydrate Chemistry and Biochemistry, vol. 30, pp. 227256, 1974.

[32] O. Zaragoza, M. A. Blazquez, and C. Gancedo, "Disruption of the Candida albicans TPS1 Gene encoding trehalose-6phosphate synthase impairs formation of hyphae and decreases infectivity," Journal of Bacteriology, vol. 180, no. 15, pp. 3809-3815, 1998.

[33] T. Mahmud, Methods of Producing Validamycin A Analogs and Uses Thereof, U.S. Patent No. 8,101,732, 2012.

[34] T. Iwasa, H. Yamamoto, and M. Shibata, "Studies on validamycins, new antibiotics. I. Streptomyces hygroscopicus var. limoneus nov. var., validamycin-producing organism," The Japanese Journal of Antibiotics, vol. 23, no. 6, pp. 595-602, 1970.

[35] T. Iwasa, E. Higashide, H. Yamamoto, and M. Shibata, "Studies on validamycins, new antibiotics. II," The Journal of Antibiotics, vol. 24, no. 2, pp. 107-113, 1971.

[36] T. Mahmud, S. Lee, and H. G. Floss, "The biosynthesis of acarbose and validamycin," The Chemical Record, vol. 1, no. 4, pp. 300-310, 2001.

[37] H. Dong, T. Mahmud, I. Tornus, S. Lee, and H. G. Floss, "Biosynthesis of the validamycins: identification of intermediates in the biosynthesis of validamycin A by Streptomyceshygroscopicus var.limoneus," Journal of the American Chemical Society, vol. 123, no. 12, pp. 2733-2742, 2001.

[38] G. D. Robson, P. J. Kuhn, and A. P. J. Trinci, "Effects of validamycin A on the morphology, growth and sporulation of Rhizoctonia cerealis, Fusarium culmorum and other fungi," Microbiology, vol. 134, no. 12, pp. 3187-3194, 1988.

[39] H. S. Randhawa, A. Chowdhary, K. Preeti Sinha, T. Kowshik, and V. K. Vijayan, "Evaluation of peptone glucose fluconazole agar as a selective medium for rapid and enhanced isolation of Aspergillus fumigatus from the respiratory tract of bronchopulmonary aspergillosis patients colonized by Candida albicans," Medical Mycology, vol. 44, no. 4, pp. 343-348, 2006.

[40] B. De Pauw, T. J. Walsh, J. P. Donnelly et al., "Revised definitions of invasive fungal disease from the European organization for research and treatment of cancer/invasive fungal infections cooperative group and the national institute of allergy and infectious diseases mycoses study group (EORTC/ MSG) consensus group," Clinical Infectious Diseases, vol. 46, no. 12, pp. 1813-1821, 2008.

[41] J. P. Donnelly, "Revision and update of the consensus definitions of invasive fungal disease from the European organization for research and treatment of cancer and the mycoses study group education and research consortium," Clinical Infectious Diseases, 2019.

[42] A. Thammahong, "Aspergillus fumigatus trehalose-regulatory subunit homolog moonlights to mediate cell wall homeostasis through modulation of chitin synthase activity," MBio, vol. 8, no. 2, 2017.

[43] G. J. Fischer, "Lipoxygenase activity accelerates programmed spore germination in Aspergillus fumigatus," Frontiers in Microbiology, vol. 8, p. 831, 2017.

[44] N. Grahl, T. M. Dinamarco, S. D. Willger, G. H. Goldman, and R. A. Cramer, "Aspergillus fumigatus mitochondrial electron transport chain mediates oxidative stress homeostasis, hypoxia responses and fungal pathogenesis," Molecular Microbiology, vol. 84, no. 2, pp. 383-399, 2012.

[45] W. W. J. van de Sande, M. Tavakol, W. van Vianen, and I. A. J. M. Bakker-Woudenberg, "The effects of antifungal agents to conidial and hyphal forms of Aspergillus fumigatus," Medical Mycology, vol. 48, no. 1, pp. 48-55, 2010.

[46] K. M. Shepardson, L. Y. Ngo, V. Aimanianda et al., "Hypoxia enhances innate immune activation to Aspergillus fumigatus through cell wall modulation," Microbes and Infection, vol. 15, no. 4, pp. 259-269, 2013.

[47] A. Thammahong, "An Ssd1 homolog impacts trehalose and chitin biosynthesis and contributes to virulence in Aspergillus fumigatus," mSphere, vol. 4, no. 3, 2019.

[48] Clinical and Laboratory Standards Institute, Reference Method for Broth Dilution Antifungal Susceptibility Testing of Filamentous Fungi, CLSI document M38-A3, Wayne, PA, USA, 2017.

[49] J. Meletiadis, S. Pournaras, E. Roilides, and T. J. Walsh, "Defining fractional inhibitory concentration index cutoffs for additive interactions based on self-drug additive combinations, Monte Carlo simulation analysis, and in vitro-in vivo correlation data for antifungal drug combinations against Aspergillus fumigatus," Antimicrobial Agents and Chemotherapy, vol. 54, no. 2, pp. 602-609, 2010.

[50] Y.-h. Park, D. Kim, J. Dai, and Z. Zhang, "Human bronchial epithelial BEAS-2B cells, an appropriate in vitro model to study heavy metals induced carcinogenesis," Toxicology and Applied Pharmacology, vol. 287, no. 3, pp. 240-245, 2015.

[51] T.-C. Zhou, B.-G. Kim, and J.-J. Zhong, "Enhanced production of validamycin A in Streptomyces hygroscopicus 5008 by engineering validamycin biosynthetic gene cluster," $A p$ plied Microbiology and Biotechnology, vol. 98, no. 18, pp. 7911-7922, 2014.

[52] Q. Wu, "Omics for understanding synergistic action of validamycin A and Trichoderma asperellum GDFS1009 against maize sheath blight pathogen," Science Reports, vol. 7, p. 40140, 2017.

[53] J. Muller, T. Boller, and A. Wiemken, "Effects of validamycinA, a potent trehalase inhibitor, and phytohormones on trehalose metabolism in roots and root-nodules of soybean and cowpea," Planta, vol. 197, no. 2, pp. 362-368, 1995.

[54] B. Tang, M. Yang, Q. Shen, Y. Xu, H. Wang, and S. Wang, "Suppressing the activity of trehalase with validamycin disrupts the trehalose and chitin biosynthesis pathways in the rice brown planthopper, Nilaparvata lugens," Pesticide Biochemistry and Physiology, vol. 137, pp. 81-90, 2017.

[55] N. Tatun, O. Wangsantitham, J. Tungjitwitayakul, and S. Sakurai, "Trehalase activity in fungus-growing termite, Odontotermes feae (Isoptera: termitideae) and inhibitory effect of validamycin," Journal of Economic Entomology, vol. 107, no. 3, pp. 1224-1232, 2014. 
[56] M. Yamaguchi, H.-J. Park, S. Ishizuka, K. Omata, and M. Hirama, "Chemistry and antimicrobial activity of caryoynencin analogs," Journal of Medicinal Chemistry, vol. 38, no. 26, pp. 5015-5022, 1995.

[57] N. Asano, T. Yamaguchi, Y. Kameda, and K. Matsui, "Effect of validamycins on glycohydrolases of Rhizoctonia solani," The Journal of Antibiotics, vol. 40, no. 4, pp. 526-532, 1987.

[58] J. P. Guirao-Abad, "Analysis of validamycin as a potential antifungal compound against Candida albicans," International Microbiology, vol. 16, no. 4, pp. 217-225, 2013.

[59] J. M. Thevelein, "Regulation of trehalose mobilization in fungi," Microbiological Reviews, vol. 48, no. 1, pp. 42-59, 1984.

[60] G. J. G. Ruijter, M. Bax, H. Patel et al., "Mannitol is required for stress tolerance in Aspergillus niger conidiospores," Eukaryotic Cell, vol. 2, no. 4, pp. 690-698, 2003.

[61] M. R. van Leeuwen, P. Krijgsheld, R. Bleichrodt et al., "Germination of conidia of Aspergillus niger is accompanied by major changes in RNA profiles," Studies in Mycology, vol. 74, no. 1, pp. 59-70, 2013.

[62] F. Barchiesi, E. Spreghini, M. Sanguinetti et al., "Effects of amphotericin B on Aspergillus flavus clinical isolates with variable susceptibilities to the polyene in an experimental model of systemic aspergillosis," Journal of Antimicrobial Chemotherapy, vol. 68, no. 11, pp. 2587-2591, 2013.

[63] D. Brodmann, A. Schuller, J. Ludwig-Müller et al., "Induction of trehalase in Arabidopsis plants infected with the trehaloseproducing pathogen Plasmodiophora brassicae," Molecular Plant-Microbe Interactions, vol. 15, no. 7, pp. 693-700, 2002.

[64] J. Müller, R. A. Aeschbacher, A. Wingler, T. Boller, and A. Wiemken, "Trehalose and trehalase in Arabidopsis," Plant Physiology, vol. 125, no. 2, pp. 1086-1093, 2001.

[65] A. Barraza, G. Estrada-Navarrete, M. E. Rodriguez-Alegria et al., "Down-regulation of PvTRE1enhances nodule biomass and bacteroid number in the common bean," New Phytologist, vol. 197, no. 1, pp. 194-206, 2013.

[66] J. Chen, "Different functions of the insect soluble and membrane-bound trehalase genes in chitin biosynthesis revealed by RNA interference," PLoS One, vol. 5, no. 4, Article ID e10133, 2010.

[67] M. López, N. A. Tejera, and C. Lluch, "Validamycin A improves the response of Medicago truncatula plants to salt stress by inducing trehalose accumulation in the root nodules," Journal of Plant Physiology, vol. 166, no. 11, pp. 1218-1222, 2009.

[68] H. Merzendorfer and L. Zimoch, "Chitin metabolism in insects: structure, function and regulation of chitin synthases and chitinases," The Journal of Experimental Biology, vol. 206, no. Pt 24, pp. 4393-4412, 2003.

[69] M. J. Paul, L. F. Primavesi, D. Jhurreea, and Y. Zhang, "Trehalose metabolism and signaling," Annual Review of Plant Biology, vol. 59, no. 1, pp. 417-441, 2008.

[70] J. Müeller, T. Boller, and A. Wiemken, "Effects of validamycin A, a potent trehalase inhibitor, and phytohormones on trehalose metabolism in roots and root nodules of soybean and cowpea," Planta, vol. 197, no. 2, pp. 362-368, 1995.

[71] K. Seo, H. Akiyoshi, and Y. Ohnishi, "Alteration of cell wall composition leads to amphotericin B resistance in Aspergillus flavus," Microbiology and Immunology, vol. 43, no. 11, pp. 1017-1025, 1999.

[72] A. K. Caffrey-Carr, "Interleukin lalpha is critical for resistance against highly virulent Aspergillus fumigatus isolates," Infection and Immunity, vol. 85, no. 12, 2017.
[73] N. Amarsaikhan, "Isolate-dependent growth, virulence, and cell wall composition in the human pathogen Aspergillus fumigatus," PLoS One, vol. 9, no. 6, Article ID e100430, 2014. 